\title{
Immunohistochemistry for human concentrative nucleoside transporter 3 protein predicts fludarabine sensitivity in chronic lymphocytic leukemia
}

\author{
Roger Y Tsang ${ }^{1,2}$, Cheryl Santos ${ }^{1,3}$, Sunita Ghosh ${ }^{1}$, Laith Dabbagh ${ }^{4}$, Karen King ${ }^{1,2}$, \\ James Young ${ }^{5}$, Carol E Cass ${ }^{1,3}$, John R Mackey ${ }^{1,2}$ and Raymond Lai ${ }^{4,6}$ \\ ${ }^{1}$ Department of Oncology, University of Alberta, Edmonton, AB, Canada; ${ }^{2}$ Department of Medical Oncology, \\ Cross Cancer Institute, Edmonton, AB, Canada; ${ }^{3}$ Department of Experimental Oncology, Cross Cancer \\ Institute, Edmonton, AB, Canada; ${ }^{4}$ Department of Laboratory Medicine and Pathology, Cross Cancer \\ Institute, Edmonton, AB, Canada; ${ }^{5}$ Department of Physiology, University of Alberta, Edmonton, AB, Canada \\ and ${ }^{6}$ Department of Laboratory Medicine and Pathology, University of Alberta, Edmonton, AB, Canada
}

\begin{abstract}
Fludarabine (F-ara-A) is widely used as palliative treatment in chronic lymphocytic leukemia (CLL). Clinical resistance is frequently observed, and adverse effects are common. To date, no practical assay exists to identify patients likely to derive benefit from F-ara-A. We previously reported that high mRNA levels encoding human concentrative nucleoside transporter 3 (hCNT3) protein in CLL correlated with clinical resistance to F-ara-A. This study explores the value of immunohistochemistry (IHC) for hCNT3 as a marker of F-ara-A resistance in CLL. We studied 36 CLL patients who received F-ara-A monotherapy and had suitable pre-F-ara-A tissue available. IHC was performed with validated hCNT3-specific monoclonal antibodies and quantitatively scored by a hematopathologist blinded to clinical outcomes. Relationships between hCNT3 staining in CLL cells and time to progression (TTP), overall response (OR), and overall survival (OS) were assessed. Dichotomization of quantitative hCNT3 staining showed that subjects with high hCNT3 IHC scores had a significantly shorter TTP with F-ara-A treatment compared to those with a low score (hazard ratio, HR, 3.16; $P=0.006$ ). Median TTP was 4.7 vs $\mathbf{1 1 . 2}$ months, respectively. On multivariate analysis, hCNT3 score was the only clinical parameter independently associated with TTP (HR, 3.12; $P=0.01$ ). OR and OS did not differ significantly between the dichotomized groups. We found a strong relationship between IHC staining of hCNT3 and clinical resistance to F-ara-A therapy in CLL. If confirmed, IHC for hCNT3 may be routinely used to predict those patients unlikely to benefit from F-ara-A, thereby avoiding F-ara-A-related toxicities.
\end{abstract}

Modern Pathology (2008) 21, 1387-1393; doi:10.1038/modpathol.2008.110; published online 4 July 2008

Keywords: chronic lymphocytic leukemia; nucleoside analogue; fludarabine; hCNT3; immunohistochemistry; drug sensitivity

Chronic lymphocytic leukemia (CLL) is the most common hematological malignancy in Western countries, affecting adults with a median age of 70 years at diagnosis. ${ }^{1}$ In the United States, it accounts for at least $30 \%$ of all leukemias, ${ }^{1}$ with estimates of 15340 new cases and 4500 deaths in the year 2007 alone. ${ }^{2}$ Although different chemotherapeutic agents are used in the treatment of this indolent lymphoid malignancy, none have been shown to be superior in terms of survival advantage..$^{3,4}$

Correspondence: Dr R Lai, MD, PhD, Department of Laboratory Medicine and Pathology, Cross Cancer Institute, 11560 University Avenue, Edmonton, AB, Canada T6G 1Z2.

E-mail: raymondl@cancerboard.ab.ca

Received 14 March 2008; revised 15 May 2008; accepted 18 May 2008; published online 4 July 2008
The most active single agent in CLL is the purine nucleoside analog fludarabine (9- $\beta$-D-arabinofuranosyl-2-fluoroadenine, or F-ara-A). Clinical studies of F-ara-A monotherapy in CLL have demonstrated it to be at least as effective as alkylator agent therapies, yielding responses in $63-79 \%$ of untreated patients and $32-48 \%$ of pretreated patients. ${ }^{5-8} \mathrm{~A}$ recent Cochrane Database meta-analysis of five randomized controlled trials in patients with previously untreated CLL identified significantly increased overall response (OR) and complete response rates, and longer progression-free survival using single agent purine nucleoside analogs compared with standard alkylating agent-based regimens. ${ }^{4}$

Clinical resistance to F-ara-A is an important issue, and is frequently observed. Resistance to F-ara-A may occur by several mechanisms, 
including reduced drug uptake and intracellular drug accumulation. ${ }^{9}$ Patients receiving $\mathrm{F}$-ara-A, especially older patients, ${ }^{10}$ are at risk for potentially serious complications, including myelosuppression, ${ }^{11-14}$ increased risk of WHO grades III/IV infections, ${ }^{4,13}$ prolonged immunosuppression, ${ }^{12,13,15-17}$ and hemolytic anemia. ${ }^{4,18-21}$ There is currently no practical assay available to predict which CLL patients are most likely to derive benefit from F-ara-A.

Fludarabine requires protein-mediated transport for cellular entry and cytotoxicity. In humans, these transporters include two members of the equilibrative nucleoside transporter (ENT) family (hENT1/2), and three members of the concentrative nucleoside transporter (CNT) family (hCNT1/2/3). We previously reported that high mRNA levels of human concentrative nucleoside transporter 3 (hCNT3) in pre-F-ara-A treatment CLL samples correlated with clinical resistance to F-ara-A, with a shorter time to progression (TTP; hazard ratio, HR, 4.67; $P<0.01$ ) and decreased OR (11 vs 69\%, $P=0.002) .{ }^{22}$ To further evaluate the importance of hCNT3 as a routine clinical predictive marker, in the present study we explored the value of immunohistochemistry (IHC) for the hCNT3 protein as a marker of F-ara-A resistance in CLL.

\section{Materials and methods}

\section{Patient Selection}

Patients who received F-ara-A for any clinical indication, from 1 November 1999 to 4 July 2005 at the Cross Cancer Institute (CCI) in Edmonton, AB, Canada, were retrospectively identified through computerized pharmacy records. Those with a pathologically confirmed diagnosis of CLL were included provided that all the following criteria were met: (1) no previous F-ara-A treatment prior to tissue sampling; (2) received F-ara-A as monotherapy; and (3) available tissue (lymph node, spleen, liver, bone marrow) suitable for immunohistochemical assessment. Computerized and manual chart review was performed to obtain key clinical parameters, including age, gender, Rai stage ${ }^{23}$ white blood cell count (WBC), absolute lymphocyte count, lactate dehydrogenase level (LDH), Eastern Cooperative Oncology Group (ECOG) performance status score, ${ }^{24}$ line of F-ara-A chemotherapy, clinical treatment response, and hematological treatment response. Full human ethics approval was obtained through the Research Ethics Review Board at the CCI.

\section{Evaluation of Response to Fludarabine}

The 1996 National Cancer Institute Working Group (NCI/WG) criteria for $\mathrm{CLL}^{25}$ were used for evaluating response to F-ara-A therapy. The only exception was the bone marrow biopsy criterion for defining complete remission, because of the retrospective nature of the study.

Complete remission was defined by the presence of all the following being present for 2 or more months: absence of symptoms attributable to CLL, normal findings on physical examination, absolute lymphocyte count $<4000$ per $\mu$ l, absolute neutrophil count $>1500$ per $\mu$ l, platelet count $>100000$ per $\mu$, and untransfused hemoglobin concentration $>11 \mathrm{~g}$ per $100 \mathrm{ml}$.

Partial response was defined by the presence of all the following for 2 or more months: a reduction in previously enlarged nodes, spleen, and liver by at least $50 \%$, and absolute neutrophil count $\geq 1500$ per $\mu \mathrm{l}$, or platelet count $\geq 100000$ per $\mu \mathrm{l}$, or hemoglobin concentration $\geq 11 \mathrm{~g}$ per $100 \mathrm{ml}$, or $50 \%$ improvement over pretherapy reductions in hemoglobin concentration and/or platelet count.

Progressive disease was defined by the presence of one or more of the following: an increase of $50 \%$ or more in the size of nodes, spleen, liver, or the appearance of new nodal involvement; an increase of $50 \%$ or more in the total lymphocyte count to at least 5000 per $\mu \mathrm{l}$; transformation to a more aggressive histology such as Richter's syndrome.

A complete OR was defined as both a complete hematological response and a complete clinical response. A partial OR was defined as at least a partial clinical response and at least a partial hematological response, but not a complete OR.

\section{Immunohistochemistry of hCNT3 Protein in Banked CLL Tissue}

An immunohistochemical assay to detect hCNT3 in clinical samples of CLL was developed using monoclonal antibodies raised against a synthetic hCNT3 peptide (conjugated to keyhole limpet hemocyanin) that corresponded to amino-acid residues 45-69 of the predicted intracellular loop near the $\mathrm{N}$ terminus of the protein. The origin and validation of these monoclonal anti-hCNT3 antibodies, named TL3360, are described elsewhere. ${ }^{26}$ Goat anti-mouse antibodies and horseradish peroxidase-labeled dextran polymer (DAKO EnVision +) were from DAKO Corporation (Carpinteria, CA, USA). All other reagents were of analytical grade and commercially available.

Formalin-fixed paraffin-embedded blocks of preF-ara-A treatment archival tissue were obtained from the CCI. Tissue sections of $4-6 \mu \mathrm{m}$ thickness were dried in an oven at $59^{\circ} \mathrm{C}$ for $2 \mathrm{~h}$. Sections were rehydrated through three changes of xylene, graded ethanol from 100 to $50 \%$, and then water ( 10 min per change). Slides were microwaved in a rapid microwave histoprocessor (RHS Milestone, Bergamo, Italy) at a controlled final temperature of $100^{\circ} \mathrm{C}$ for $10 \mathrm{~min}$ in DAKO TRIS buffer ( $\mathrm{pH}$ 9.9), cooled for $6 \mathrm{~min}$, and washed in running cold tap water. Slides were incubated in $3 \% \mathrm{H}_{2} \mathrm{O}_{2}$ in methanol for $10 \mathrm{~min}$, 
and rewashed for $5 \mathrm{~min}$ in tap water. Tissue sections were then incubated with 1:5 dilutions of the TL3360 anti-hCNT3 antibody preparations in a room temperature humidity chamber for $30 \mathrm{~min}$. The sections were then rinsed in PBS $(\mathrm{pH} 7.2)$ and immersed in PBS for 5 min, incubated with Envision (DAKO EnVision +) goat anti-mouse dextran conjugate for $30 \mathrm{~min}$, washed in PBS for $5 \mathrm{~min}$, incubated with diaminobenzidene solution, rinsed, counter-stained with hematoxylin, dehydrated through graded alcohols and xylene, and coverslipped. Negative controls were provided by either omitting the TL3360 primary antibodies, by using antibody preparations from which anti-hCNT3 antibodies had been removed by incubation with an excess of anti-hCNT3 peptide, or by substitution of primary antibodies with anti-CD38 monoclonal antibodies. Positive controls were provided by sections of pancreas, a tissue known to express high levels of hCNT3 message ${ }^{27}$ and shown to have readily detectible membrane hCNT3 staining by IHC. Quantitative scoring using light microscopy was performed by a hematopathologist (RL) blinded to clinical characteristics and outcomes. Immunohistochemical scoring of hCNT3 protein was assigned a score from 0 to 3 based on staining intensity with homogeneous staining in greater than $50 \%$ of the cell population: no staining $=0$; weakly positive $=1$; moderately positive $=2$; and strongly positive $=3$.

\section{Primary and Secondary Outcomes}

Time to progression, which was calculated from the initiation date of F-ara-A therapy until the date of disease progression, was chosen as the primary end point of this study because it could be determined more reliably than the objective response rate. For those patients surviving without disease progression, TTP was censored at the time of last follow-up. Overall survival (OS) and OR rates were chosen as secondary outcomes.

Univariate and multivariate analyses for TTP and OS were performed using standard statistical techniques, including the Kaplan-Meier method, logrank test, and Cox proportional hazards model. Statistical significance was set at a $P$-value $<0.1$ at the univariate level, and a $P$-value $<0.05$ at the multivariate level. The $\chi^{2}$-method was used to analyze OR rates. All statistical analyses were performed using SAS version 9.1.3 software (SAS Institute Inc., Cary, NC, USA).

\section{Results}

\section{Baseline Patient Characteristics and Treatment Response}

In total, 374 patients received F-ara-A at the CCI for any clinical indication from 1 November 1999 to 4
Table 1 Baseline patient characteristics

\begin{tabular}{|c|c|c|}
\hline & At diagnosis & $\begin{array}{c}\text { At fludarabine } \\
\text { initiation }\end{array}$ \\
\hline Patients no. & 36 & 36 \\
\hline Male & 25 & 25 \\
\hline Female & 11 & 11 \\
\hline \multicolumn{3}{|l|}{ Age, years } \\
\hline Median (range) & $62.5(37-81)$ & $66.5(37-85)$ \\
\hline \multicolumn{3}{|l|}{ CLL Rai stage (no.) } \\
\hline Stage 0 & 8 & 0 \\
\hline Stage I & 21 & 8 \\
\hline Stage II & 5 & 13 \\
\hline Stage III & 0 & 5 \\
\hline Stage IV & 2 & 10 \\
\hline \multicolumn{3}{|c|}{ WBC count, $\times 10^{9}$ per liter ${ }^{\mathrm{a}}$} \\
\hline Median (range) & $15.4(4.1-178.2)$ & $86(4-248.4)$ \\
\hline Unknown no. & 3 & 0 \\
\hline \multicolumn{3}{|c|}{ Lymphocyte count, $\times 10^{9}$ per liter $^{\mathrm{b}}$} \\
\hline Median (range) & $12.6(1.5-178.2)$ & $79.3(1.1-238.5)$ \\
\hline Unknown no. & 3 & 1 \\
\hline \multicolumn{3}{|c|}{$L D H$ value, $I U$ per liter ${ }^{c}$} \\
\hline Median (range) & $472(171-754)$ & $545(391-1754)$ \\
\hline Unknown no. & 17 & 17 \\
\hline \multicolumn{3}{|c|}{ ECOG performance status score (no.) } \\
\hline 0 & Not applicable & 2 \\
\hline 1 & Not applicable & 26 \\
\hline 2 & Not applicable & 7 \\
\hline 3 or 4 & Not applicable & 1 \\
\hline \multicolumn{3}{|c|}{$\begin{array}{l}\text { ECOG, Eastern Cooperative Oncology Group; LDH, Lactate dehydrogenase; } \\
\text { WBC, white blood cell. }\end{array}$} \\
\hline \multicolumn{3}{|c|}{${ }^{\mathrm{a}}$ Normal range for $\mathrm{WBC}$ count $=4-11 \times 10^{9}$ per liter. } \\
\hline \multicolumn{3}{|c|}{${ }^{b}$ Normal range for lymphocyte count $=0.8-4.4 \times 10^{9}$ per liter. } \\
\hline
\end{tabular}

July 2005. Of those, 51 patients with complete clinical data and who received F-ara-A as treatment for CLL were identified. Of those, 9 patients had either only post-F-ara-A treatment tissue available or unavailable tissue and were therefore excluded from the study. Of the remaining 42 cases, 6 patients received F-ara-A as combination therapy (F-ara-A/ cyclophosphamide, or F-ara-A/cyclophosphamide/ rituximab) rather than as monotherapy and were therefore excluded. Ultimately, 36 patients with CLL who received F-ara-A monotherapy, with suitable pre-F-ara-A treatment tissue available, were included in our study.

Baseline patient characteristics at times of CLL diagnosis and F-ara-A initiation are summarized in Table 1. Performance status at time of F-ara-A initiation, as measured by the ECOG score, consisted of two patients with score 0,26 with score 1 , seven with score 2 , and one with score 3 or 4 . The median number of F-ara-A cycles received was 3 (range, $1-8)$. Eight patients $(22 \%)$ received six or more cycles. In total, 19 patients received F-ara-A by the intravenous route, 16 by the oral route, and one by 

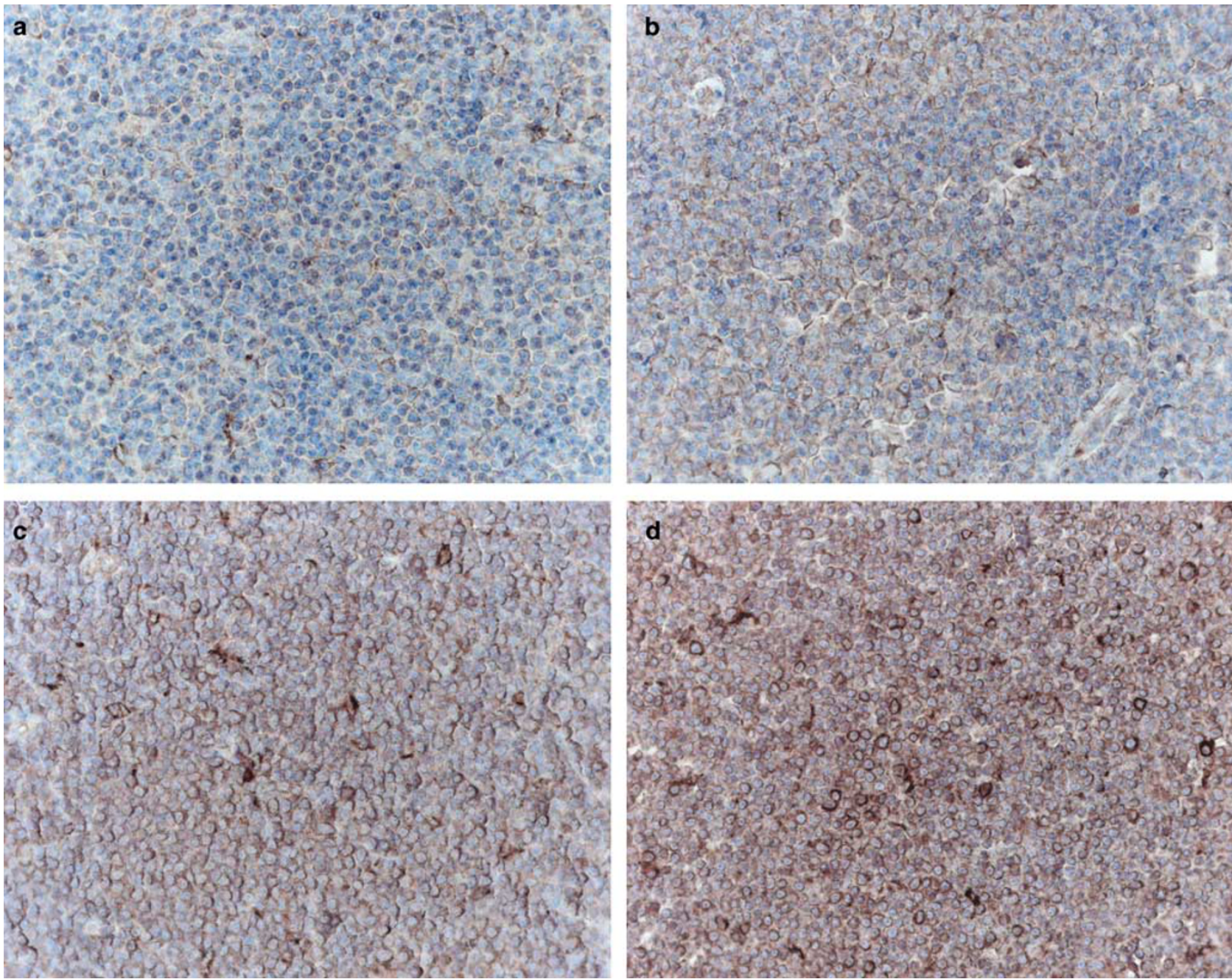

Figure 1 Representative photomicrographs of hCNT3 immunohistochemistry in pre-fludarabine treatment CLL tissue. (a) Score of 0; (b) score of 1; (c) score of 2; (d) score of 3. Photomicrographs were taken with a Zeiss Axiocam camera attached to a Zeiss Axioskop2 Plus microscope. A Zeiss Fluar $\times 40$ objective lens with 1.3 numerical aperture was used. Images were acquired using Zeiss Axiovision version 3.1 software (Carl Zeiss MicroImaging GmbH, Germany).

both routes. The median dose of F-ara-A administered was $710 \mathrm{mg}$ (range, 200-2000) by the intravenous route, $715 \mathrm{mg}$ (range, 200-2000) by the oral route, and $1725 \mathrm{mg}$ by the intravenous and oral routes. In total, 12 patients received F-ara-A as firstline chemotherapy for CLL, 20 as second-line chemotherapy, and four as third-line chemotherapy. For those receiving second or third-line chemotherapy, the alkylating agent chlorambucil was uniformly used in the prior treatments.

Complete hematological responses were observed in seven patients $(19 \%)$, partial hematological responses in 15 patients (42\%), and no hematological responses in 14 patients (39\%). Complete clinical responses were observed in 11 patients $(31 \%)$, partial clinical responses in 13 patients $(36 \%)$, and no clinical responses in 12 patients $(33 \%)$. Complete ORs to F-ara-A monotherapy were observed in 6 patients (17\%), partial ORs in 14 patients $(39 \%)$, and no responses in 16 patients $(44 \%)$.

\section{Quantitative IHC Scoring of hCNT3 Protein}

Immunohistochemical staining of hCNT3 protein in pre-F-ara-A treatment samples was quantitatively scored on a 0-3 scale as described earlier. Of the 36 cases, 5 had a score of 0,12 had a score of 1,18 had a score of 2 , and 1 had a score of 3 . Representative photomicrographs are illustrated in Figure 1.

\section{Relationships between hCNT3 IHC Score and Clinical Outcomes}

The CLL population was dichotomized by quantitative hCNT3 staining (scores 0 and 1 vs 2 and 3). The relationship between the dichotomized groups and TTP is shown in Figure 2. Subjects with high hCNT3 IHC scores (scores 2 and 3 ) had a significantly shorter TTP with F-ara-A treatment compared to those with low hCNT3 IHC scores (scores 0 and 1), with a median TTP of 4.7 vs 11.2 months, respectively, and a HR of $3.16(95 \% \mathrm{CI}=1.39-7.17$, 


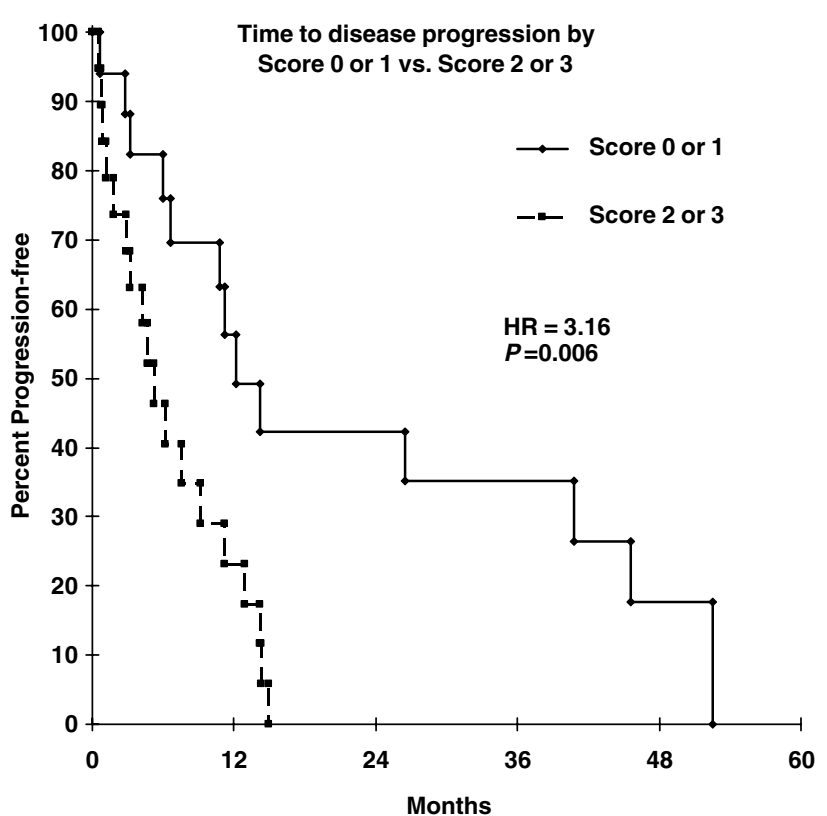

Figure 2 Time to progression, dichotomized by hCNT3 immunostaining.

$P=0.006$ ) on univariate analysis. Rai stage and line of F-ara-A treatment were also statistically significant at the univariate level (HR, 2.56; 95\% $\mathrm{CI}=0.94-6.96$; and $P=0.06$ for Rai stage II with stage I as reference; $\mathrm{HR}, 0.35 ; 95 \% \mathrm{CI}=0.11-1.18$; $P=0.09$ for third-line F-ara-A therapy with first-line as reference). On multivariate analysis, only the hCNT3 IHC score was independently associated with TTP, with a HR of $3.12(95 \% \mathrm{CI}=1.26-7.73$, $P=0.01$ ). OR rates, as shown in Table 2 , were $42 \%$ in subjects with high hCNT3 IHC scores (scores 2 and 3), compared with $71 \%$ in those with low hCNT3 IHC scores (scores 0 and 1), with $\chi^{2}=2.95$ and $P=0.08$. OS (not shown) did not differ significantly between the dichotomized groups $(P=0.15)$.

\section{Discussion}

Chronic lymphocytic leukemia is a common, indolent lymphoid malignancy for which no curative treatment currently exists. In the absence of significant patient symptoms, signs, or laboratory abnormalities, many patients are placed on a 'waitand-watch' policy given that standard chemotherapy has not yet been shown to confer a survival advantage. ${ }^{4}$ Although several independent prognostic factors, ${ }^{28}$ including CD-38, ${ }^{29}$ ZAP-70, ${ }^{30,31} \beta-2$ microglobulin, ${ }^{32}$ chromosomal abnormalities, ${ }^{29}$ including deletions of $17 \mathrm{p}$ or $11 \mathrm{q}$ as detected by FISH, and immunoglobulin Vh genes ${ }^{29}$ have been identified to discriminate CLL patient populations between favorable and less favorable prognostic groups, the majority of chemotherapeutic decisions
Table 2 Overall response, dichotomized by hCNT3 immunostaining

\begin{tabular}{lcc}
\hline & Score 0 or 1 & Score 2 or 3 \\
\hline $\begin{array}{l}\text { Progressive or stable } \\
\text { disease (no.) }\end{array}$ & 5 & 11 \\
$\begin{array}{l}\text { Partial or complete } \\
\text { response (no.) }\end{array}$ & 12 & 8 \\
$\begin{array}{l}\text { Overall response } \\
\text { rate (\%) }\end{array}$ & 71 & 42 \\
& & $\begin{array}{l}\chi^{2}=2.95 \\
P=0.08\end{array}$ \\
& & \\
\hline
\end{tabular}

are still based upon prognostic groups defined by the Binet et $a l^{33}$ or $\mathrm{Rai}^{23}$ staging systems, rather than a validated predictive assay. ${ }^{34,35}$ A recent study found that patients with high-risk cytogenic features such as deletions of $17 p$ or $11 q$ were associated with reduced progression-free survival, and whom should be considered for non-F-ara-A-based therapies. ${ }^{36}$

Fludarabine is a nucleoside analog commonly used in the clinical setting as palliative monotherapy for CLL, but it is not an innocuous drug and has potential serious adverse effects, including myelosuppression and prolonged immunosuppression. To date, there are no readily available predictive assays to identify the patient population most likely to benefit from F-ara-A treatment. Although an assay employing ex vivo differential staining cytotoxicity $^{37}$ has been used to identify F-ara-A-resistant CLL patient populations, ${ }^{38-40}$ this assay is not widely utilized due to its requirement for purification of CLL cells and 48-h F-ara-A exposures. As we previously reported that high mRNA levels of hCNT3 in pre-F-ara-A treatment CLL samples correlated with clinical resistance to F-ara-A, we undertook this study to further evaluate the importance of IHC for the hCNT3 protein as a routinely applicable clinical predictive marker.

Our results showed a strong and independent relationship between IHC staining of the hCNT3 protein and clinical resistance to F-ara-A therapy in CLL. With the CLL population dichotomized by quantitative hCNT3 staining, there was a significantly shorter TTP in subjects with high hCNT3 IHC scores, with a median TTP of 4.7 months vs a median TTP of 11.2 months in subjects with low hCNT3 IHC scores. Age, WBC count, lymphocyte count, LDH value, and ECOG performance status score were not statistically significant variables at the univariate level. On multivariate analysis incorporating Rai stage and line of F-ara-A treatment, hCNT3 IHC score was the only clinicopathological factor independently associated with TTP, a clinically relevant outcome, with a HR of 3.12 and $P$-value of 0.01 . These results are consistent in magnitude and direction with our previous report. ${ }^{22}$ In terms of OR rates, there was a trend toward lower 
response rates in subjects with high hCNT3 IHC scores, but this did not reach statistical significance given the small sample size. As expected, there was no difference in OS between the dichotomized groups, taking into account that standard chemotherapy has not been shown to confer a survival advantage.

On the basis of these findings, we therefore infer that hCNT3 protein has a direct mechanistic role in cellular resistance to F-ara-A, although the underlying mechanisms are not yet fully understood. Although F-ara-A resistance mechanisms may include reduced drug uptake and intracellular drug accumulation, ${ }^{9}$ paradoxically we found that it was high, rather than low, hCNT3 IHC staining that correlated with shorter TTP and clinical resistance. Possible mechanisms include: (1) the regulated coordination of the hCNT3 gene with other genes that indirectly mediate F-ara-A resistance; (2) high hCNT3 expression is a biological marker of a distinct CLL subgroup with intrinsic F-ara-A resistance; and (3) hCNT3 protein localization to intracellular organelles such as the mitochondria may directly modulate F-ara-A toxicity by augmenting physiologic nucleoside uptake.

Although our results show promise that IHC for the hCNT3 protein may be of value as a practical assay to predict which CLL patients are most likely to derive benefit from F-ara-A therapy, our study has some limitations. These include the retrospective nature of the study, relatively small numbers of patients, prior treatment with chlorambucil that might modulate hCNT3 protein abundance and localization, the analysis of heterogeneous tissues, as well as potential intra- and interobserver variabilities in IHC quantitative scoring. In addition, with the exclusion of patients who received F-ara-A as part of combination therapy, it becomes difficult to predict whether our results can be extrapolated to this different population, especially as F-ara-Abased combination therapy becomes more widely used in younger patients and as part of clinical trials. Although the route of F-ara-A administration varied between intravenous and oral, the median dose was similar (710 vs $715 \mathrm{mg}$, respectively) however, and previous studies have established similar clinical efficacy between the two routes. ${ }^{41,42}$

Our study demonstrates the potential value of adding IHC for the hCNT3 protein to the routine diagnostic IHC evaluation of CLL as a predictive marker in the setting of F-ara-A monotherapy. Larger, multicenter prospective studies are warranted to further validate these observations, including assessment of intra- and interobserver variabilities associated with IHC evaluation. It would also be of great interest to include studies of established prognostic markers, such as ZAP-70 and immunoglobulin Vh mutation status, for correlation with hCNT3. If confirmed, high hCNT3 expression detected by IHC may identify F-ara-A-resistant patients who should pursue non-F-ara-A therapies, thereby avoiding F-ara-A-related toxicities, and would lend further support to attempts to modulate hCNT3 activity to circumvent F-ara-A resistance.

\section{Acknowledgement}

This work was supported by grants from the National Cancer Institute of Canada, Alberta Cancer Board, and Alberta Cancer Foundation. We thank Tony Reiman for his helpful review of the paper. RYT contributed to study design, collected clinical data, analyzed and interpreted data, performed initial statistical analysis, and drafted the paper; CS contributed to immunohistochemical analysis; SG contributed to the final statistical analysis; LD assisted in immunohistochemical methods development; KK, JY, and CEC contributed new reagents and analytical tools; JRM initiated this study, analyzed and interpreted data, and performed initial statistical analysis; RL contributed to study design, performed the pathology reviews, and analyzed and interpreted data.

\section{Disclosure/conflict of interest}

The authors declare no competing financial or other conflicts of interest.

\section{References}

1 Hernandez JA, Land KJ, McKenna RW. Leukemias, myeloma, and other lymphoreticular neoplasms. Cancer 1995;75:381-394.

2 Jemal A, Siegel R, Ward E, et al. Cancer statistics, 2007. CA Cancer J Clin 2007;57:43-66.

3 Eichhorst BF, Busch R, Hopfinger G, et al. Fludarabine plus cyclophosphamide vs fludarabine alone in first-line therapy of younger patients with chronic lymphocytic leukemia. Blood 2006;107:885-891.

4 Steurer M, Pall G, Richards S, et al. Purine antagonists for chronic lymphocytic leukaemia. Cochrane Database Syst Rev 2006;3:CD004270.

5 Johnson S, Smith AG, Loffler H, et al. Multicentre prospective randomised trial of fludarabine vs cyclophosphamide, doxorubicin, and prednisone (CAP) for treatment of advanced-stage chronic lymphocytic leukaemia. The French Cooperative Group on CLL. Lancet 1996;347:1432-1438.

6 Sorensen JM, Vena DA, Fallavollita A, et al. Treatment of refractory chronic lymphocytic leukemia with fludarabine phosphate via the group $\mathrm{C}$ protocol mechanism of the National Cancer Institute: five-year follow-up report. J Clin Oncol 1997;15:458-465.

7 Rai KR, Peterson BL, Appelbaum FR, et al. Fludarabine compared with chlorambucil as primary therapy for chronic lymphocytic leukemia. N Engl J Med 2000; 343:1750-1757.

8 Leporrier M, Chevret S, Cazin B, et al. Randomized comparison of fludarabine, CAP, and ChOP in 938 previously untreated stage $\mathrm{B}$ and $\mathrm{C}$ chronic lymphocytic leukemia patients. Blood 2001;98:2319-2325. 
9 Galmarini CM, Mackey JR, Dumontet C. Nucleoside analogues: mechanisms of drug resistance and reversal strategies. Leukemia 2001;15:875-890.

10 Robak T. Therapy of chronic lymphocytic leukaemia with purine nucleoside analogues: facts and controversies. Drugs Aging 2005;22:983-1012.

11 Keating MJ, Estey E, O’Brien S, et al. Clinical experience with fludarabine in leukaemia. Drugs 1994;47(Suppl 6):39-49.

12 Cheson BD. Infectious and immunosuppressive complications of purine analog therapy. J Clin Oncol 1995; 13:2431-2448.

13 Fenchel K, Bergmann L, Wijermans P, et al. Clinical experience with fludarabine and its immunosuppressive effects in pretreated chronic lymphocytic leukemias and low-grade lymphomas. Leuk Lymphoma 1995;18:485-492.

14 Polizzotto MN, Tam CS, Milner A, et al. The influence of increasing age on the deliverability and toxicity of fludarabine-based combination chemotherapy regimens in patients with indolent lymphoproliferative disorders. Cancer 2006;107:773-780.

15 Bergmann L, Fenchel K, Jahn B, et al. Immunosuppressive effects and clinical response of fludarabine in refractory chronic lymphocytic leukemia. Ann Oncol 1993;4:371-375.

16 Anaissie EJ, Kontoyiannis DP, O’Brien S, et al. Infections in patients with chronic lymphocytic leukemia treated with fludarabine. Ann Intern Med 1998;129:559-566.

17 Samonis G, Kontoyiannis DP. Infectious complications of purine analog therapy. Curr Opin Infect Dis 2001;14:409-413.

18 Myint H, Copplestone JA, Orchard J, et al. Fludarabine-related autoimmune haemolytic anaemia in patients with chronic lymphocytic leukaemia. Br J Haematol 1995;91:341-344.

19 Gonzalez H, Leblond V, Azar N, et al. Severe autoimmune hemolytic anemia in eight patients treated with fludarabine. Hematol Cell Ther 1998;40: 113-118.

20 Taha HM, Narasihman P, Venkatesh L, et al. Fludarabine-related hemolytic anemia in chronic lymphocytic leukemia and lymphoproliferative disorders. Am J Hematol 1998;59:316.

21 Weiss RB, Freiman J, Kweder SL, et al. Hemolytic anemia after fludarabine therapy for chronic lymphocytic leukemia. J Clin Oncol 1998;16:1885-1889.

22 Mackey JR, Galmarini CM, Graham KA, et al. Quantitative analysis of nucleoside transporter and metabolism gene expression in chronic lymphocytic leukemia (CLL): identification of fludarabine-sensitive and insensitive populations. Blood 2005;105:767-774.

23 Rai KR, Sawitsky A, Cronkite EP, et al. Clinical staging of chronic lymphocytic leukemia. Blood 1975;46:219-234.

24 Oken MM, Creech RH, Tormey DC, et al. Toxicity and response criteria of the Eastern Cooperative Oncology Group. Am J Clin Oncol 1982;5:649-655.

25 Cheson BD, Bennett JM, Grever M, et al. National Cancer Institute-sponsored Working Group guidelines for chronic lymphocytic leukemia: revised guidelines for diagnosis and treatment. Blood 1996;87:4990-4997.

26 Lang TT. Studies of Recombinant and Native Human Concentrative Nucleoside Transporters and Their Role in Nucleoside Pharmacology and Adenosine Signaling. University of Alberta: Edmonton, 2003.

27 Ritzel MW, Ng AM, Yao SY, et al. Molecular identification and characterization of novel human and mouse concentrative Na+-nucleoside cotransporter proteins (hCNT3 and mCNT3) broadly selective for purine and pyrimidine nucleosides (system cib). J Biol Chem 2001;276:2914-2927.

28 Rai KR, Wasil T, Iqbal U, et al. Clinical staging and prognostic markers in chronic lymphocytic leukemia. Hematol Oncol Clin North Am 2004;18:795-805, vii.

29 Krober A, Seiler T, Benner A, et al. V(H) mutation status, CD38 expression level, genomic aberrations, and survival in chronic lymphocytic leukemia. Blood 2002;100:1410-1416.

30 Orchard JA, Ibbotson RE, Davis Z, et al. ZAP-70 expression and prognosis in chronic lymphocytic leukaemia. Lancet 2004;363:105-111.

31 Del Principe MI, Del Poeta G, Buccisano F, et al. Clinical significance of ZAP-70 protein expression in B-cell chronic lymphocytic leukemia. Blood 2006; 108:853-861.

32 Fayad L, Keating MJ, Reuben JM, et al. Interleukin-6 and interleukin-10 levels in chronic lymphocytic leukemia: correlation with phenotypic characteristics and outcome. Blood 2001;97:256-263.

33 Binet JL, Auquier A, Dighiero G, et al. A new prognostic classification of chronic lymphocytic leukemia derived from a multivariate survival analysis. Cancer 1981;48:198-206.

34 Rozman C, Montserrat E. Chronic lymphocytic leukemia. N Engl J Med 1995;333:1052-1057.

35 Binet JL, Caligaris-Cappio F, Catovsky D, et al. Perspectives on the use of new diagnostic tools in the treatment of chronic lymphocytic leukemia. Blood 2006;107:859-861.

36 Grever MR, Lucas DM, Dewald GW, et al. Comprehensive assessment of genetic and molecular features predicting outcome in patients with chronic lymphocytic leukemia: results from the US Intergroup Phase III Trial E2997. J Clin Oncol 2007;25:799-804.

37 Weisenthal LM, Marsden JA, Dill PL, et al. A novel dye exclusion method for testing in vitro chemosensitivity of human tumors. Cancer Res 1983;43:749-757.

38 Bosanquet AG, Bell PB. Enhanced ex vivo drug sensitivity testing of chronic lymphocytic leukaemia using refined DiSC assay methodology. Leuk Res 1996;20:143-153.

39 Bosanquet AG, Johnson SA, Richards SM. Prognosis for fludarabine therapy of chronic lymphocytic leukaemia based on ex vivo drug response by DiSC assay. Br J Haematol 1999;106:71-77.

40 Mason JM, Drummond MF, Bosanquet AG, et al. The DiSC assay. A cost-effective guide to treatment for chronic lymphocytic leukemia? Int J Technol Assess Health Care 1999;15:173-184.

41 Boogaerts MA, Van Hoof A, Catovsky D, et al. Activity of oral fludarabine phosphate in previously treated chronic lymphocytic leukemia. J Clin Oncol 2001;19:4252-4258.

42 Rossi JF, van Hoof A, de Boeck $\mathrm{K}$, et al. Efficacy and safety of oral fludarabine phosphate in previously untreated patients with chronic lymphocytic leukemia. J Clin Oncol 2004;22:1260-1267. 